OPEN ACCESS

Edited by:

Arghya Paul,

Western University, Canada

Reviewed by:

Corinne A. Hoesli,

McGill University, Canada Hasmukh A. Patel,

Aramco Services Company,

United States

*Correspondence:

Eric M. Brey

eric.brey@utsa.edu

Specialty section:

This article was submitted to

Polymer Chemistry,

a section of the journal

Frontiers in Chemistry

Received: 23 June 2020

Accepted: 21 October 2020

Published: 12 November 2020

Citation:

Somo SI, Brown JM and Brey EM (2020) Dual Crosslinking of Alginate

Outer Layer Increases Stability of

Encapsulation System.

Front. Chem. 8:575278.

doi: 10.3389/fchem.2020.575278

\section{Dual Crosslinking of Alginate Outer Layer Increases Stability of Encapsulation System}

\author{
Sami I. Somo ${ }^{1}$, Jacob M. Brown ${ }^{2}$ and Eric M. Brey ${ }^{2 *}$ \\ ${ }^{1}$ Biomedical Engineering Department, Illinois Institute of Technology, Chicago, IL, United States, ${ }^{2}$ Biomedical Engineering \\ Department, University of Texas at San Antonio, San Antonio, TX, United States
}

The current standard treatment for Type 1 diabetes is the administration of exogenous insulin to manage blood glucose levels. Cellular therapies are in development to address this dependency and allow patients to produce their own insulin. Studies have shown that viable, functional allogenic islets can be encapsulated inside alginate-based materials as a potential treatment for Type 1 diabetes. The capability of these grafts is limited by several factors, among which is the stability and longevity of the encapsulating material in vivo. Previous studies have shown that multilayer Alginate-Poly-L-Ornithine-Alginate (A-PLO-A) microbeads are effective in maintaining cellular function in vivo. This study expands upon the existing encapsulation material by investigating whether covalent crosslinking of the outer alginate layer increases stability. The alginate comprising the outer layer was methacrylated, allowing it to be covalently crosslinked. Microbeads with a crosslinked outer layer exhibited a consistent outer layer thickness and increased stability when exposed to chelating agents in vitro. The outer layer was maintained in vivo even in the presence of a robust inflammatory response. The results demonstrate a technique for generating A-PLO-A with a covalently crosslinked outer layer.

Keywords: alginate (PubChem CID: 91666324), islets, crosslinking, type 1 diabetes (or diabetes), encapsulation

\section{INTRODUCTION}

Diabetes mellitus is a metabolic disease characterized by defective insulin secretion by pancreatic $\beta$-cells in response to glucose stimulation. The current pharmacological treatment of Type I diabetes involves administration of exogenous insulin in response to blood glucose levels. Glucose levels are either self-monitored or tested by an implanted device. While implanted systems have dramatically improved in recent years, a level of control equivalent to endogenous insulin secretion from $\beta$-cells cannot be achieved (Scharp and Marchetti, 2014; Kollmer et al., 2016; Dinnyes et al., 2020). Over time, this can lead to complications such as kidney failure, loss of vision, foot ulcers, and limb amputation (Litwak et al., 2013).

Cell-based therapies have been proposed as an alternative to exogenous insulin therapy. Endocrine $\beta$-cell clusters, islets, within the pancreas have been implanted into patients to restore normal pancreatic function. Despite promising results achieved with naked islets, the lack of available donor tissue, the number of islets needed per patient, the need for immunosuppressants, and eventual loss of $\beta$-cell function over time has hampered islet transplantation. Investigation of biomaterials for the encapsulation and immunoisolation of islets is a strategy to protect these cells from the host autoimmune system and increase their longevity. 
Alginate has been investigated extensively as an encapsulation medium for $\beta$-cell islets. These microencapsulation systems have been shown to improve the survival and function of islet grafts implanted in both genetically and chemically induced diabetic animal models (Omer et al., 2003; Qi et al., 2008; Rengifo et al., 2014; Lawandi et al., 2015; Vegas et al., 2016). In clinical trials, these systems have enabled some patients to reduce their exogeneous insulin usage for extended periods of time. The efficacy and duration of the improvement varied, however, with some patients requiring multiple transplants and none becoming completely insulin independent. The cause of the inconsistency in patient outcomes is hard to discern, as it might arise from the use of varying alginate compositions, islet sources, implantation sites, and/or patient to patient variation (Calafiore et al., 2005; Kollmer et al., 2016). While the primary reason for graft failure is unknown, one identified mechanism is the breakdown of the alginate microbeads following implantation. Alginate microbeads which are only ionically crosslinked can become unstable due to calcium chelation and sodium ion exchange (van Raamsdonk et al., 2002; Strand et al., 2017). Breakdown of ionically crosslinked beads has been observed in in vivo models (Ibarra et al., 2016).

Alginate is relatively stable biomaterial, but breakdown can occur due to a number of factors, including inflammation or mechanical stress (Figure 1). Implanting alginate microbeads in the body triggers the foreign body response, which ultimately leads to the growth and differentiation of immune cells and fibroblasts around the microbeads. This fibrous capsule has

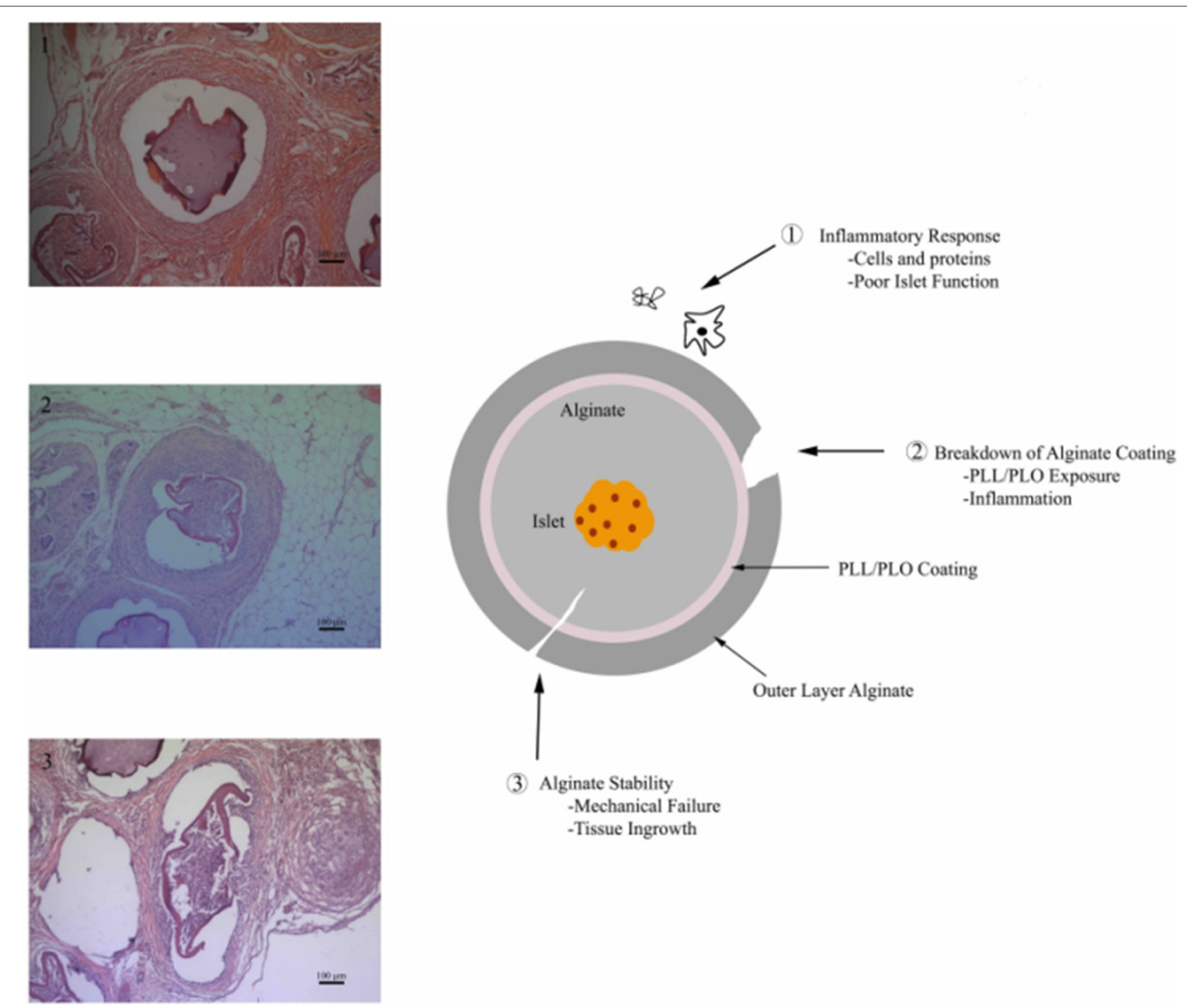

FIGURE 1 | Alginate microbead failure mechanisms (1) robust inflammatory response leading to encapsulation of the material, (2) failure or breakdown of alginate coating exposing the underlying positively charged permselective membrane resulting in a chronic inflammatory response, and (3) alginate breakdown via chemical degradation or mechanical failure. 
the potential to not only disrupt the transport of nutrients, compromising the function and survival of the islets, but can also directly lead to the degradation of the microbeads (Anderson et al., 2008; Veiseh et al., 2015). The collection of inflammatory cells on the surface of the bead may also lead to the degradation of the alginate crosslinks by increasing reactive oxygen species production (de Vos et al., 2003a). These effects may be modulated by adjusting the size, shape, and polycation layer concentration of the microbeads, among other attributes (Ponce et al., 2006; Veiseh et al., 2015). The implant can also be subjected to different mechanical stresses depending on where it is located. Beads implanted within the peritoneal cavity experience different mechanical stresses compared to those implanted within the omentum (Dufrane et al., 2006). Increasing stability of the microbeads and their resistance to the inflammatory response could increase their effective duration when applied as a therapy for diabetes.

Alginate microbeads currently used for $\beta$-cell encapsulation typically consist of three separate individual layers. The first is an inner layer alginate core containing $\beta$-cell islets (Somo et al., 2017). The second layer is a semipermeable monolayer which allows oxygen and nutrients to diffuse in and out, while preventing the encapsulated cells from being exposed to larger molecules or other cells. Finally, this permselective layer is covered with a second alginate layer (ranging from a single monolayer to $\sim 100 \mu \mathrm{m}$ thickness) to prevent cell interactions with the polycationic semipermeable membrane, which can lead to increased inflammation. The outer layer can also be used to deliver molecules that locally modulate the inflammatory response angiogenesis (Khanna et al., 2010, 2013). Dual layer alginate microbeads with an intermediate Poly-Lornithine microbeads, referred to here as A-PLO-A (AlginatePoly-L-Ornithine-Alginate), can protect islets and enable longterm function (Kollmer et al., 2016). However, any breakdown of the outer alginate layer may lead to failure of the implants.

Several investigators have introduced covalent crosslinks into alginate-based materials to increase stability (Hall et al., 2011; Breger et al., 2015; Hillberg et al., 2015). For example, methacrylated single-layer alginate microbeads were shown to enhance stability of microspheres in vitro and in vivo when used to dual crosslink alginate microbeads, without affecting cell viability (Somo et al., 2018). However, A-PLO-A microbeads were not used in these studies. In addition, the alginate used for the studies was a crude alginate solution which is poorly characterized and has limitations regarding its interaction with the polycationic membrane (Thu et al., 1996; Bhujbal et al., 2014). In addition, previous studies have not focused on stabilizing the outer layer, which is the interface between the biomaterial and the host, playing a significant role in modulating the inflammatory response.

In this study, the dual (simultaneously ionically and covalently) crosslinking of the outer alginate layer was investigated as a method for increasing the stability of multilayer A-PLO-A microcapsules. The in vivo portion of the study was performed without encapsulating cells to reduce the risk of complications, as the primary intent was to compare differing outer layer compositions, which would not contain cells in the final application. The effect of the photopolymerization process on MIN6 cells, routinely encapsulated using these systems, was assessed as part of a previous study. Their viability was maintained above $90 \%$ at methacrylation efficiencies of up to $4 \%$ (Somo et al., 2018). Methacrylated low viscosity ultra-pure sodium alginate with high guluronic acid content (LVG) was used as the alginate base for the outer layer. Methacrylation allowed for the covalent crosslinking of the LVG alginate, in addition to the normally ionically crosslinked mechanism. A-PLO-A microbeads with a methacrylated LVG outer layer exhibited controlled outer layer thickness $(\sim 170 \mu \mathrm{m})$ and increased stability when exposed to $\mathrm{CaCl}_{2}$ chelating agents. The outer layer was maintained in vivo even in the presence of a robust inflammatory response. These results demonstrate that A-PLO-A microbeads with a covalently crosslinked outer layer exhibit increased stability.

\section{MATERIALS AND METHODS}

\section{Materials}

Low viscosity (20-200 $\mathrm{mPaS})$ ultra-pure sodium alginate with high mannuronic (LVM) and high guluronic acid (LVG) contents were purchased from Nova-Matrix $(75-200 \mathrm{kDa}, \mathrm{G} / \mathrm{M}$ ratios of 1 and 1.5, respectively). 2-hydroxy-2-methylpropiophenone (Irgacure 1173), poly-l-ornithine (PLO) hydrochloride (MW: 15,000-30,000), 2-morpholinoethanesulfonice acid (MES), N-Hydroxysuccinimide (NHS), 1-ethyl-3-(3dimethylaminopropyl)-carbodiimide hydrochloride (EDC), lipopolysaccharide (LPS) from Escherichia coli 0111:B4, Dulbecco's Modified Eagle's medium (DMEM), Dulbecco's phosphate-buffered saline (DPBS), and 2-mercaptoethanol were purchased from Sigma-Aldrich (St. Louis, MO). Fetal Bovine Serum (FBS) and penicillin-streptomycin was purchased from Life Technologies (Waltham, MA). MIN6 cell line was purchased from AddexBio (San Diego, CA). 2-Aminoethyl methacrylate hydrochloride (AEMA) was purchased from Polysciences (Warminster, PA). Live/Dead kit was purchased from Invitrogen (Eugene, OR). Solutions for alginate microbead fabrications were made using the following chemicals: HEPES, $\mathrm{NaCl}, \mathrm{MgCl} 2$ (Fisher Scientific); $\mathrm{CaCl} 2$ (Acros).

\section{Synthesis and Characterization of LVG Methacrylated Alginate}

Methacrylated LVG alginate was synthesized based on the modification of a previous protocol (Somo et al., 2018). Briefly, $1 \% \mathrm{w} / \mathrm{v}$ LVG alginate was dissolved in a buffer consisting of $0.5 \mathrm{M} \mathrm{NaCl}$ and $50 \mathrm{mM}$ MES. NHS and EDC were added to the mixture sequentially and mixed for $5 \mathrm{~min}$. Ninety five milligram of AEMA was added to the mixture and the reaction maintained at room temperature for $24 \mathrm{~h}$. AEMA was added to the mixture of EDC and NHS in a concentration that maintained a molar ratio of NHS:EDC:AEMA equal to 1:2:1. After $24 \mathrm{~h}$, the reaction was precipitated with excess acetone using a Buchner funnel through $5 \mu \mathrm{m}$ filter paper. The product was recovered and dissolved in $50 \mathrm{~mL}$ of deionized (DI) water and precipitated again with acetone. The product was dissolved in $50 \mathrm{~mL}$ DI water and dialyzed (MWCO 3500) against DI water for 3 days. The 
methacrylated alginate solution was filtered with a $0.22 \mu \mathrm{m}$ filter and lyophilized. As a control, unmodified alginate (LVG) was processed in the same manner in the absence of AEMA. ${ }^{1} \mathrm{H}$ nuclear magnetic resonance (NMR) was performed to evaluate methacrylation. Methacrylated alginate $(15 \mathrm{mg})$ was dissolved in $1 \mathrm{~mL}$ of deuterium oxide and placed in NMR tubes. The NMR spectrum of the methacrylated alginate was recorded on a Bruker 300 Ultrashield NMR spectrometer. The methacrylation efficiency (ME) was determined as the ratio of the integrals for the methylene protons of methacrylate $(\delta 5.3-\delta 5.8 \mathrm{ppm})$ to alginate protons $(\delta 3.5-\delta 4.0 \mathrm{ppm})$ (Figure 3A).

\section{Fabrication of Multilayered Alginate Microbeads}

Microbeads were prepared under sterile conditions using a standard method of injection into a cationic crosslinking solution. LVM alginate $(1.5 \% \mathrm{w} / \mathrm{v})$ was dissolved in a solution consisting of $25 \mathrm{mM}$ HEPES, $118 \mathrm{mM} \mathrm{NaCl}, 5.6 \mathrm{mM} \mathrm{KCl}, 2.5 \mathrm{mM}$ $\mathrm{MgCl}_{2}$. The dissolved precursor was first sterilized by extrusion through a $0.22 \mu \mathrm{m}$ syringe filter. The filtered precursor was then extruded through a $1 \mathrm{~mL}$ syringe with a blunt 20-gauge needle into $15 \mathrm{~mL}$ of a crosslinking solution consisting of $100 \mathrm{mM}$ $\mathrm{CaCl}_{2}$ and $10 \mathrm{mM}$ HEPES. The beads were incubated in the crosslinking solution for $15 \mathrm{~min}$. After $15 \mathrm{~min}$, the beads were washed three times with $2 \mathrm{mM} \mathrm{CaCl}_{2}$ and saline $(0.9 \% \mathrm{NaCl})$ for $2 \mathrm{~min}$. The microbeads were transferred into a $0.1 \%(\mathrm{w} / \mathrm{v})$ solution of PLO in normal saline and rocked for $30 \mathrm{~min}$, resulting in the formation of a PLO coating. Three washes were performed with $2 \mathrm{mM} \mathrm{CaCl}_{2}$ and $0.9 \% \mathrm{NaCl}$ solution to remove residual PLO. The PLO-coated microbeads were dried and blotted with a kimwipe to remove excess water, then transferred into an alginate solution. At this point, beads were divided into two groups, one with a regular LVG alginate outer layer, and the other with a methacrylated LVG outer layer. The microbeads were incubated in the alginate solution for $40 \mathrm{~min}$, allowing the alginate time to interact with the PLO layer. Excess alginate solution was removed, and the outer layer was crosslinked in a solution of $22 \mathrm{mM} \mathrm{CaCl}_{2}$ for both groups. The crosslinking solution for the methacrylated LVG beads additionally contained $0.05 \%(\mathrm{w} / \mathrm{v})$ Irgacure 1173 (photoinitiator), and the beads were exposed to UV light for $5 \mathrm{~min}$ for photocrosslinking. Two additional washes were performed with $2 \mathrm{mM} \mathrm{CaCl} 2$ and $0.9 \% \mathrm{NaCl}$ solution to remove unbound alginate. After synthesis, the microbeads were imaged using Axiovert 200x inverted microscope (Carl Zeiss MicroImaging) with a $5 x$ objective, and the size of the outer layer quantified with AxioVision. Ten microbeads were measured per condition, and the experiments were repeated three times. A schematic of the bead synthesis steps is shown in Figure 2.

The NMR spectrum of crosslinked methacrylated alginate was obtained by manually separating the outer layer from dual layer dual crosslinked beads. Once separated, the combined outer layers were placed into an NMR tube, lyophilized, and finally rehydrated with $\mathrm{D}_{2} \mathrm{O}$ (Figure 3B).

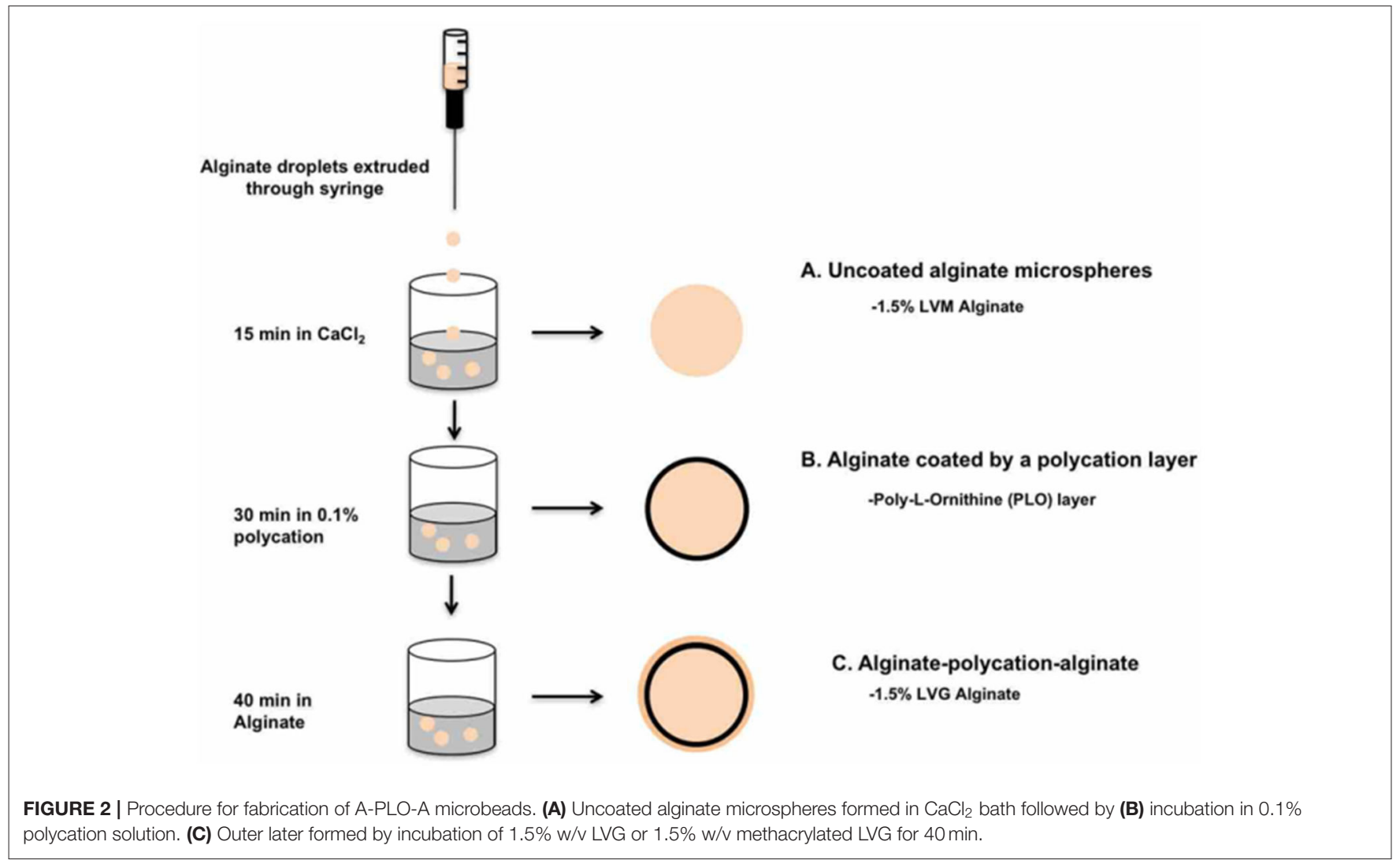




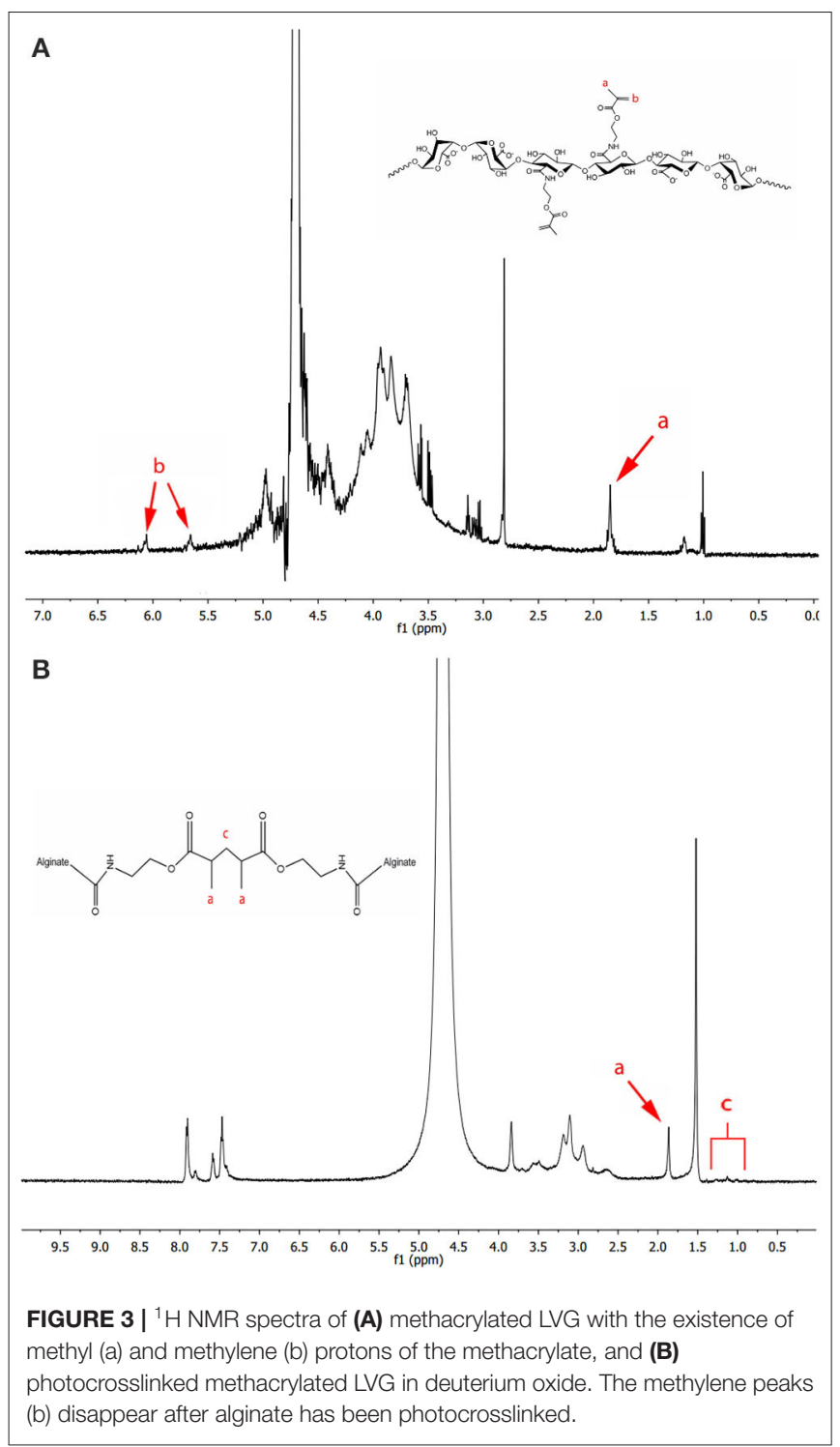

Scanning electron microscopy (SEM) was used to evaluate the surfaces of the microbeads. Alginate microbeads were incubated in $2.5 \%$ glutaraldehyde at $4{ }^{\circ} \mathrm{C}$ for $2 \mathrm{~h}$. The microbeads were then washed 3 times with distilled water. Microtubes containing the microbeads and distilled water were frozen in liquid nitrogen and then lyophilized overnight. Randomly selected microbeads were deposited on carbon conductive tape adhered to SEM aluminum stubs. The beads were analyzed using a Phenom PRO Desktop SEM (Phenom-World, Netherlands) operated at $10 \mathrm{kV}$.

\section{Stability Assessment of Outer Alginate Layer}

To assess the stability of alginate beads in vitro, microbeads were incubated in a solution consisting of $55 \mathrm{mM}$ sodium citrate, $50 \mathrm{mM} \mathrm{NaCl}$, and $30 \mathrm{mM}$ EDTA. Alginate microbeads prepared as described above were incubated for $24 \mathrm{~h}$ at $37^{\circ} \mathrm{C}$ in a humidified atmosphere. Microbeads were imaged before and after the addition of the outer layer for assessment using an Axiovert 200x inverted microscope (Carl Zeiss MicroImaging) with a $5 x$ objective. The size of the outer layer was quantified with AxioVision. To determine long term swelling properties, fresh alginate beads were incubated in saline solution at $37^{\circ} \mathrm{C}$ in a humidified atmosphere over a period of 40 days. The media was replaced after the beads were imaged. Four points along the edge of the inner layer were selected to generate a circle approximating the shape of the inner layer, and then the outer layer was determined in the same way. The computergenerated diameter of the outer layer was subtracted from the inner layer to determine the thickness of the outer layer $(n=15$ for each group).

\section{In vivo Stability Model}

Animal experiments were carried out using procedures approved by Illinois Institute of Technology's Institutional Animal Care and Use Committee. An omentum pouch model was used to evaluate microbeads in vivo. Two alginate conditions were examined: (1) A-PLO-A microbeads prepared under sterile conditions with an ionic-crosslinked outer layer and (2) dual crosslinked outer layer alginate microspheres. The outer layer was $1.5 \%(\mathrm{w} / \mathrm{v})$ concentration for both. A total of 16 animals were used, 4 per group per time point (1 and 3 weeks). Male Sprague Dawley rats (300-400 g, $n=4$; Envigo) were anesthetized initially with $5 \%$ isoflurane. Body temperature was maintained at $37^{\circ} \mathrm{C}$ with a heating pad, and anesthesia was maintained with a $2 \%$ isoflurane/oxygen gas mixture during the procedure. Each animal had their abdomen shaved, and skin scrubbed with isopropyl alcohol, followed by a povidone-iodine antiseptic solution. The omentum was surgically exposed by midline laparotomy. First, the skin was separated from muscle and a $\sim 2$-inch incision was made. Next, the underlying muscle was cut to expose the organs and the greater omentum was carefully pulled from the abdomen. Using 4-0 Ethilon suture, a purse-string suture was positioned around the edges of the omentum to create a pouch for the beads. Fifty alginate beads were placed on the anterior surface of the exposed omentum. LPS (100 $\mu \mathrm{l}$ of $50 \mu \mathrm{g}$ LPS dissolved in $1 \mathrm{~mL}$ saline) was directly injected onto the anterior surface of the omentum to stimulate an inflammatory challenge (Somo et al., 2018). Afterwards, the pouch was folded over and sutured to secure the beads inside. The underlying muscle and then skin were closed with 40 Ethilon suture. After surgery, the animals were allowed to recover, and were monitored closely. At each time point (week 1 and week 3 ) the omenta were explanted, fixed in formalin, and prepared for histological characterization and imaging. The tissues were processed for histology using standard methods and tissues were paraffin embedded. Samples were sectioned at 5 $\mu \mathrm{mm}$ thickness and stained with hematoxylin and eosin (H\&E) and Masson's Trichrome. Tissue sections were imaged using an Axiovert 200 inverted microscope (Carl Zeiss MicroImaging). The outer layers of the beads were observed and measured using the AxioVision software (Carl Zeiss MicroImaging), for each of five selected beads from each condition, five distances were quantified between the inner and outer layers. 


\section{Statistical Analysis}

Statistical analysis was performed using GraphPad Prism. All data are expressed as mean \pm standard deviation. In vitro data were analyzed using one-way ANOVA with a Tukey's post-test for normally distributed data. Values of $p<0.05$ were considered statistically significant.

\section{RESULTS}

\section{Methacrylation Efficiency}

Methacrylated alginate was produced by reacting LVG alginate with AEMA using NHS/EDC chemistry. The ${ }^{1} \mathrm{H}$ NMR spectra of methacrylated alginate is shown in Figure 3A. Peaks associated with AEMA can clearly be identified in the spectra. The methacrylated alginate had peaks corresponding to methylene ( $\delta 5.7$ and $\delta 6.1)$ and methyl $(\delta 1.9)$ groups that are formed by the reaction with AEMA. The procedure resulted in a methacrylation efficiency of $2.16 \% \pm 0.33$. The ${ }^{1} \mathrm{H}$ NMR of methacrylated LVG alginate after covalent crosslinking is shown in Figure 3B. After exposure to UV in the presence of an appropriate initiator, the absence of the methylene peaks in the spectrum indicates the reaction of the AEMA groups.

\section{Microbead Fabrication and Size}

Microbeads were synthesized using 1.5\% (w/v) LVM alginate for the inner core (Figure 1) and two formulations for the outer core: LVG and methacrylated LVG. A distinct alginate layer is present on the outside of the beads after incubation in the polycation bath (Figure 4A). Following incubation in LVG or methacrylated LVG alginate, the outer layer could be observed surrounding the alginate core and PLO layer (Figure 4B). The outer layer exhibited a roughness (Figure 4D) that was not observed in the PLO surface alone (Figure 4C) The size of the alginate outer layer

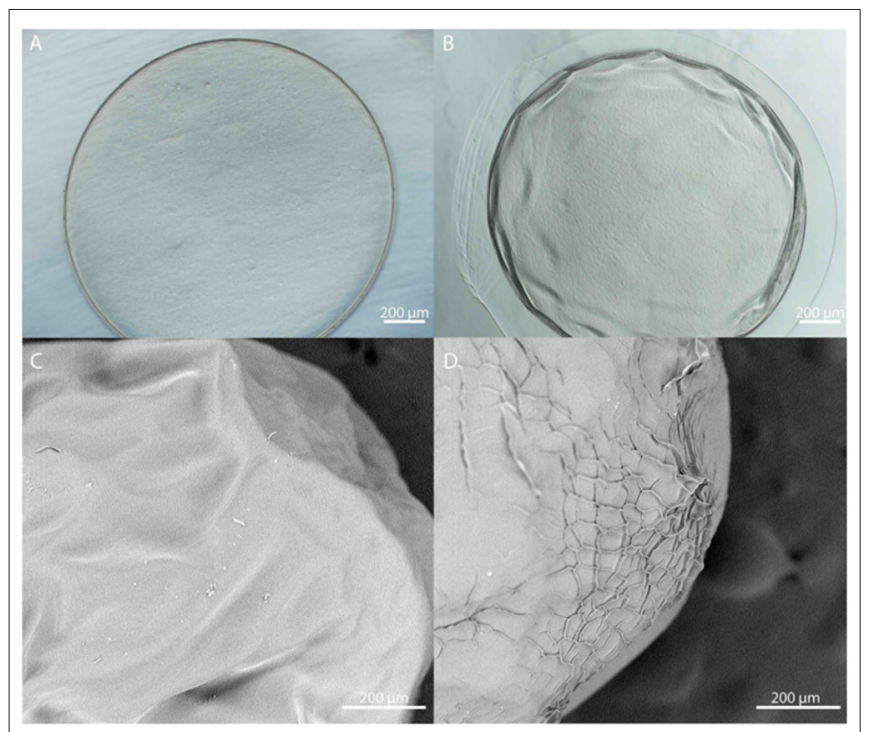

FIGURE 4 | (A,B) Phase contrast and (C,D) SEM images of PLO coated alginate microbeads $(\mathbf{A}, \mathbf{C})$ prior to and $(\mathbf{B}, \mathbf{D})$ after formation of the outer layer. did not vary significantly between the two alginate compositions or with UV exposure (Figure 5). The outer layer sizes were $179 \pm$ $25 \mu \mathrm{m}, 182 \pm 31 \mu \mathrm{m}, 167 \pm 35 \mu \mathrm{m}$ and, $168 \pm 12 \mu \mathrm{m}$ for LVG, LVG exposed to UV, methacrylated LVG, and methacrylated LVG exposed to UV, respectively $(n=10)$.

\section{Stability and Long-Term Swelling}

The stability of the alginate outer layer was first assessed in vitro based on incubation in sodium citrate, a chelating agent. A-PLOA microbeads were prepared with an LVG outer layer or a dual crosslinked methacrylated LVG outer layer and samples placed in a solution of $55 \mathrm{mM}$ sodium citrate, $50 \mathrm{mM} \mathrm{NaCl}$, and $30 \mathrm{mM}$ EDTA at $37^{\circ} \mathrm{C}$ room temperature for $4 \mathrm{~h}$ (Figure 6). The outer layer of the standard outer layer conditions (LVG) was absent after sodium citrate exposure (Figure 6A). Outer layers formed by crosslinked methacrylated LVG microbeads exhibited greater integrity without any evidence of breakdown (Figure 6D). SEM cross-sectional images of the beads are presented in Figure 7. Upon dissolution of outer layer, the smooth PLO surface is exposed, and only two distinct layers are seen, the inner alginate core and the smooth PLO layer (Figure 7B). However, the images of methacrylated LVG retained the distinct outer layer after sodium citrate exposure (Figure 7D).

The time-dependent behavior of the microbeads was evaluated by incubation in a $0.9 \% \mathrm{NaCl}$ solution $(n=17$ for each group) (Moya et al., 2012). All groups exhibited significant swelling of the outer layer within 1 day. For conditions without covalent crosslinks (LVG with and without UV exposure, methLVG without UV exposure) the size of the outer layer decreased significantly from 1 to 2 days. By day 4 the outer layer was no longer observed. In contrast, the outer layer of the covalently crosslinked outer layers (meth-LVG with UV exposure) did not vary significantly after the initial swelling for the entire duration of the study, from a maximum of $251 \pm 22 \mu \mathrm{m}$ on day 4 to 226 $\pm 33 \mu \mathrm{m}$ on day 40 . Covalent crosslinking of methacrylated LVG increased the stability of the outer later in culture (Figure 8).

\section{In vivo Stability Model}

Microbeads formed with dual crosslinked (meth-LVG exposed to UV) and control microbeads (ionic crosslinks) were evaluated

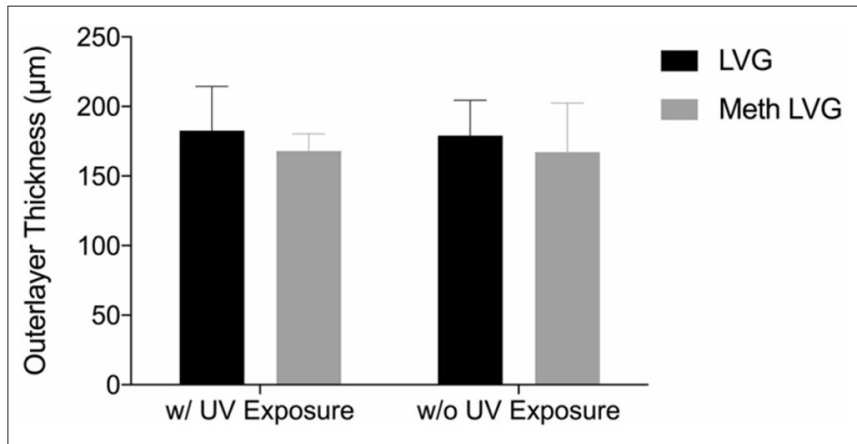

FIGURE 5 | The size of the outer layer of multilayered alginate microbeads was not statistically different with LVG and methacrylated LVG with and without UV exposure. 


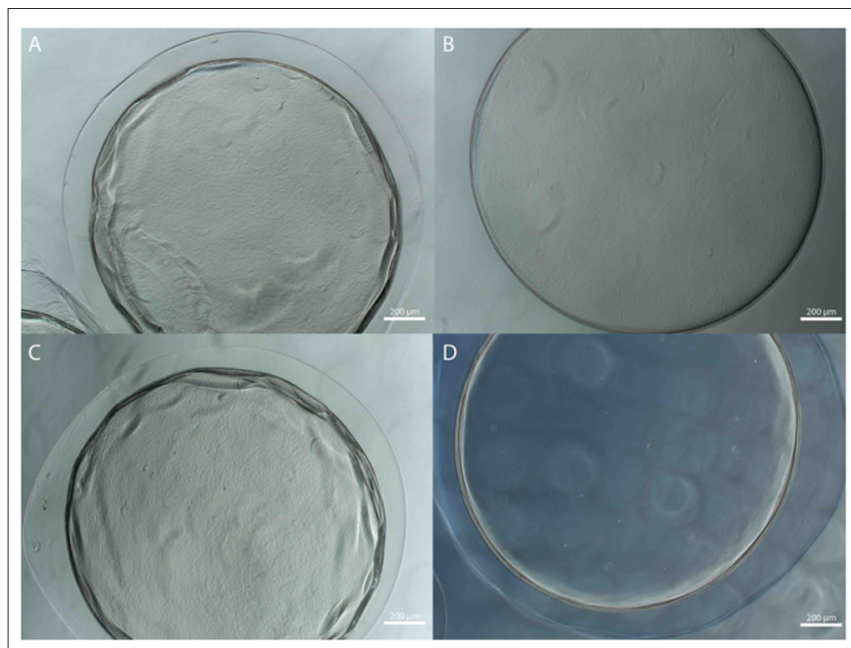

FIGURE 6 | Multilayered alginate microbeads with (A,B) LVG and (C,D) methacrylated LVG outer layer $(\mathbf{A}, \mathbf{C})$ before and $(\mathbf{B}, \mathbf{D})$ after exposure to sodium citrate.

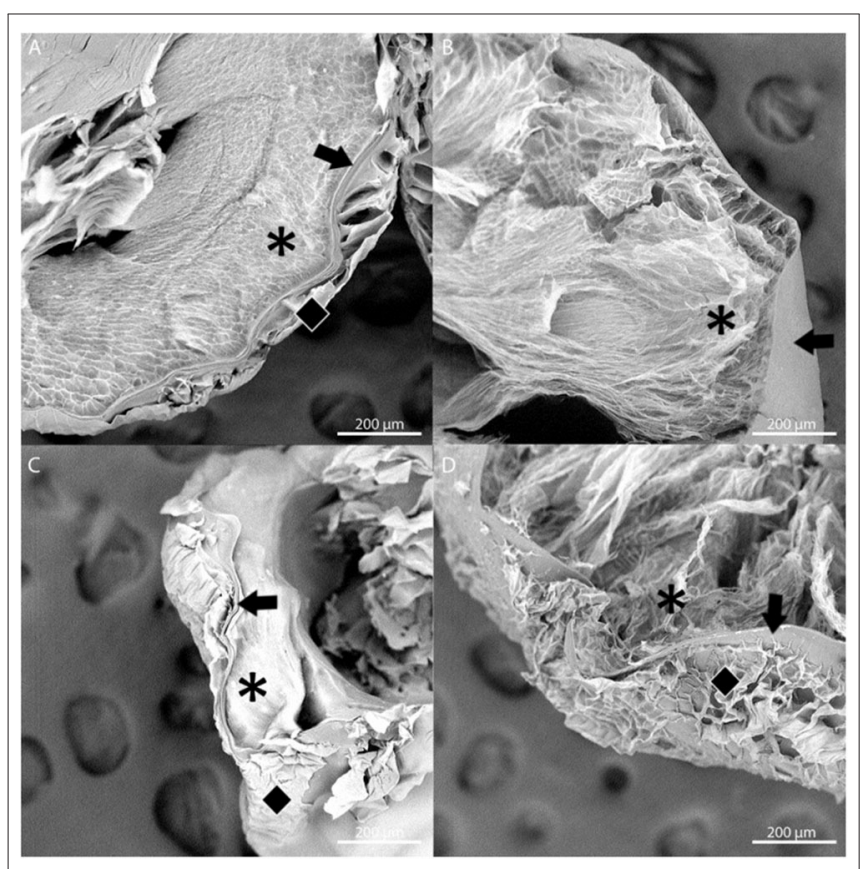

FIGURE 7 | SEM images of multilayered alginate microbeads with LVG outer layer $(\mathbf{A}, \mathbf{B})$ and methacrylated LVG outer layer $(\mathbf{C}, \mathbf{D})$ before $(\mathbf{A}, \mathbf{C})$ and after (B,D) exposure to sodium citrate for 4 hours. Layers are represented by symbols: inner core (asterisk), PLO layer (arrow), outer layer (diamond). Three distinct layers are observed with methacrylated LVG outer layer (D) after exposure to sodium citrate.

in a rat omental pouch model. During microbead implantation, $5 \mu \mathrm{g}$ LPS was applied directly to the omentum to stimulate an inflammatory response in order to challenge the stability of the alginate microbeads. This concentration was selected from previous studies because it results in a robust inflammatory response that have been shown to break down alginate-based

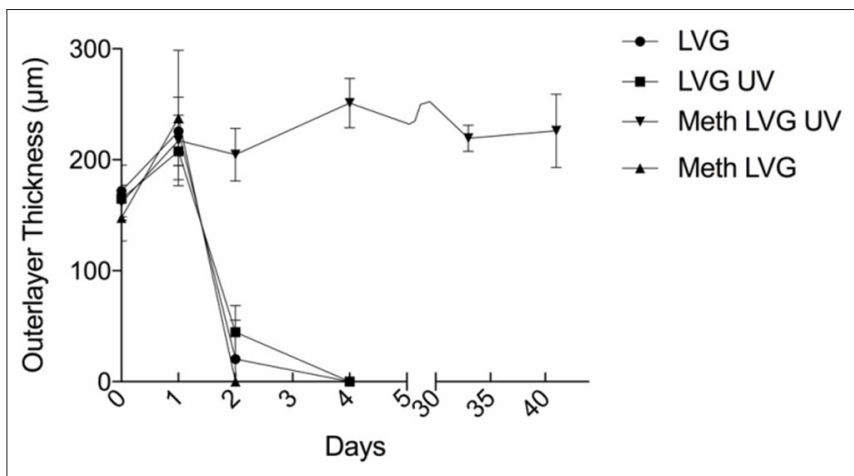

FIGURE 8 | The size of the outer layer of the microbeads vs. time during incubation in a solution of $0.9 \% \mathrm{NaCl}$ solution at $37^{\circ} \mathrm{C}$. The methacrylated LVG exposed to UV swelled initially and remained stable for over a period of 40 days.

materials without systemic toxicity (Somo et al., 2018). At weeks 1 and 3, the microbeads were harvested and processed for histological analysis. Beads of each group were observed within the pouches at harvest. H\&E and Masson's trichrome stains were performed on the samples harvested to evaluate microbead structure and inflammatory response. Alginate microbeads for both groups were observed within the tissue at both time points (Figures 9, 10). A robust inflammatory response was observed with multinucleated foreign body giant cells observed near the material surface. While histological processing can disrupt structure, the alginate microbeads were observed with the PLO layer, which appears as a thinner, dark purple line. The outer layer, which is the thicker, lighter pink section surrounding the PLO, was observed and quantified (Figure 11). The size of the outer layer at week 1 was $167 \pm 25 \mu \mathrm{m}$ and $185 \pm 38 \mu \mathrm{m}$ for LVG and methacrylated LVG, respectively. By week 3, the outer layer of the LVG beads had significantly decreased in size $(p<0.05)$ to $124 \pm 20 \mu \mathrm{m}$, but the outer layer of methacrylated LVG beads was not statistically different, with a final size of $170 \pm 35 \mu \mathrm{m}$.

\section{DISCUSSION}

The current standard treatment for diabetes is exogenous insulin, administered either manually or with a controlled pump device. Cellular therapies designed to reestablish glucose-sensitive insulin production are being developed as an alternative approach. Multilayered alginate microbeads have been investigated as a method of encapsulation to protect implanted allograft $\beta$-cell islets as a treatment for people with diabetes (de Vos et al., 2003b; Qi et al., 2008). Current literature suggests that the failure of these structures can be caused by a variety of issues, from a lack of oxygen delivery, variations in transplantation site, biocompatibility, or degradation of the encapsulation material. Several attempts have been made to increase the stability of the A-PLO-A microbeads to account for these various mechanisms of failure (Sawhney and Hubbell, 1992; Chandy et al., 1999; Eiselt et al., 1999; Lee et al., 2000; Mahou et al., 2012). This research focuses on the material 


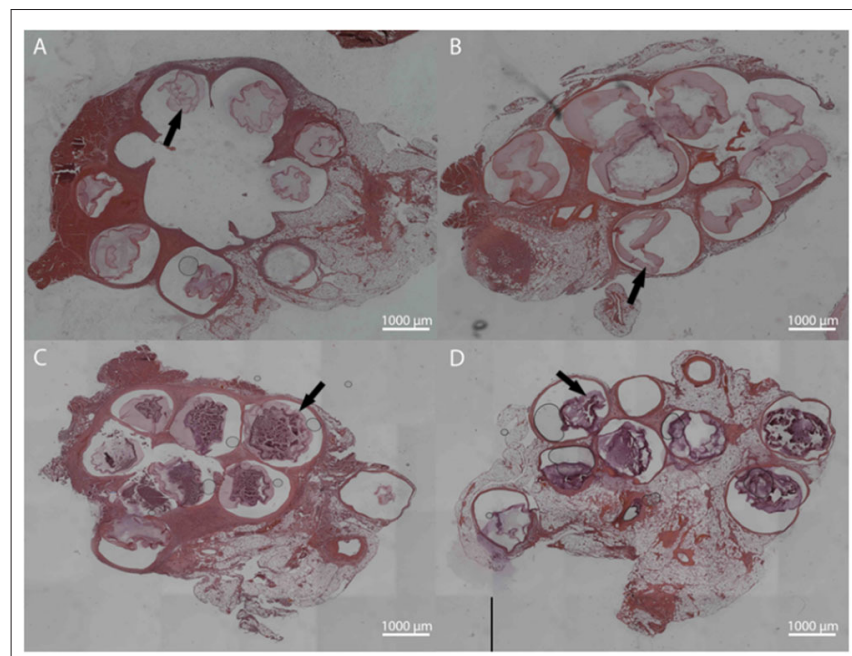

FIGURE 9 | Hematoxylin and Eosin staining for LVG $(\mathbf{A}, \mathbf{C})$ and methacrylated LVG (B,D) at 1 week (A,B) and 3 weeks (C,D). Intact microbeads observed for both groups at 1 and 3 weeks, with observable outer layer (arrows).

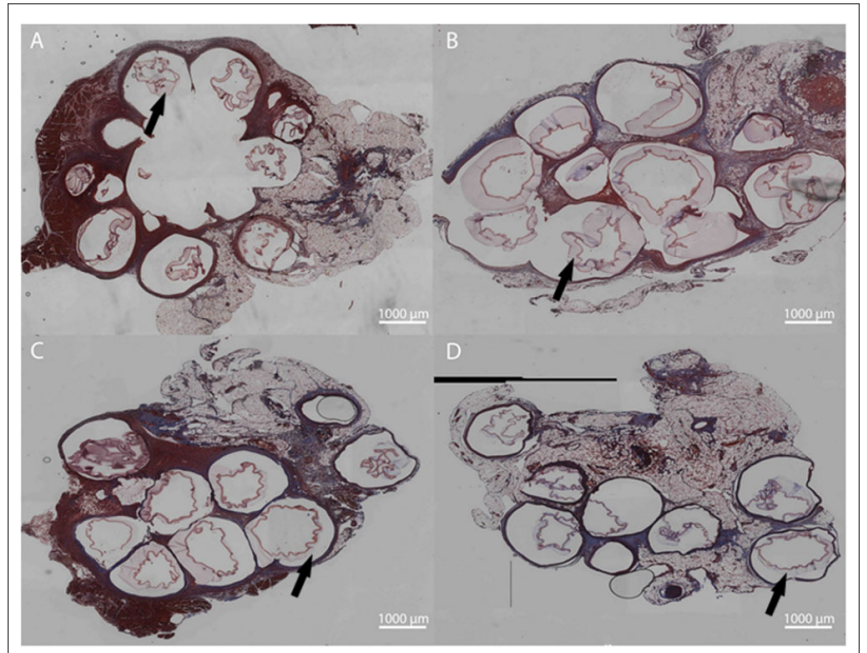

FIGURE 10 | Masson's Trichrome staining for LVG $(\mathbf{A}, \mathbf{C})$ and methacrylated LVG $(\mathbf{B}, \mathbf{D})$ at 1 week $(\mathbf{A}, \mathbf{B})$ and 3 weeks $(\mathbf{C}, \mathbf{D})$. Inflammation surrounding the microbeads are observed for both groups at all time points. Outer layers indicated with arrows.

properties of the alginate used for encapsulation or modulation of the local inflammatory response. Previous research has shown that dual crosslinking of alginate results in a more stable hydrogel even in the presence of a robust inflammatory response (Somo et al., 2018). However, the previous work focused on techniques that would enhance the stability of the inner alginate microbead. In this work methacrylated alginate was investigated as a way to produce a more stable outer layer.

In order to generate A-PLO-A microbeads with dual crosslinked outer layers, alginate was modified with AEMA to introduce methacrylate side chains. The methacrylate groups enabled the introduction of covalent crosslinks following UV

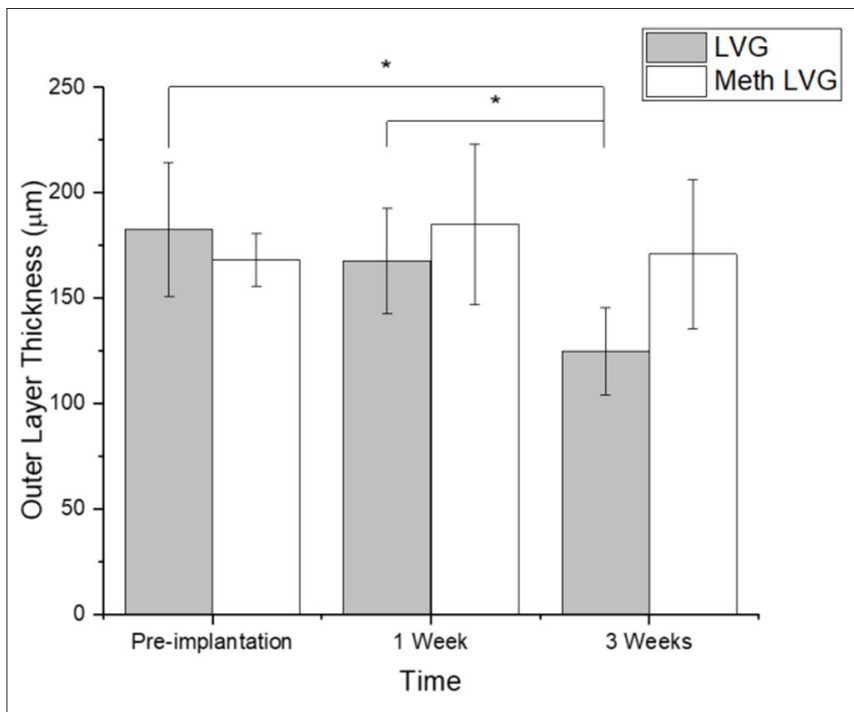

FIGURE 11 | Outer layer thickness of alginate microbeads before (pre-implant) and after in vivo stability testing ( 1 and 3 weeks). *Denotes statistical significance $(p<0.05)$.

exposure in the presence of an appropriate photoinitiator. The degree of methacrylation was kept to a minimum to allow native LVG alginate to form ionic crosslinks, which allows for an easy method for forming the outer layer. The covalent crosslinks are expected to enhance stability, as the ionic calcium crosslinks which give alginate its structure normally can be disrupted by the presence of sodium ions or other cations present in the in vivo environment. Improvement in outer layer stability may improve the overall performance of A-PLO-A microbeads, as breakdown of the outer alginate layer can result in exposure of the pro-inflammatory PLO or PLL polymer coating (Vandenbossche et al., 1993; Hall et al., 2011). Polymer rheology and biological properties of alginate-based materials depend on molecular weight. The alginate used in this study ranged from 75 to $200 \mathrm{kDa}$. With methacrylation efficiency at $2.16 \%$ the effect on molecular weight was assumed to be negligible. However, future studies will quantitatively evaluate polymer molecular weight distribution and rheology. The results of this study demonstrate the dual crosslinking of methacrylated alginate for outer layer A-PLO-A results in increased stability in vitro and in vivo.

Stability testing of methacrylated A-PLO-A microbeads showed that unlike A-PLO-A LVG microbeads with an only ionically crosslinked outer layer, the dual crosslinked alginate outer layer remained intact when exposed to calcium chelating agents. Three distinct layers are seen in SEM images of the methacrylated LVG group. The likely reason for the outer layer remaining, despite the chelation of calcium from the outer layer, are the covalent crosslinks. The chelating agent, sodium citrate, removes the calcium that initially allows for a hydrogel to form around the PLO. When the calcium is removed, the chains are soluble in aqueous solution. The methacrylated LVG contains additional covalent crosslinks that are not affected by the chelating agent. The methacrylated LVG alginate exposed to 
UV exhibited swelling comparable to other groups and remained in solution for up to 40 days.

The in vivo stability of alginate microbeads was tested based on a protocol described in previous work (Somo et al., 2018). LPS is applied directly onto the omentum at the time of implantation to test the stability of alginate systems through exposure to a sustained inflammatory challenge. This surgical method has been applied in the absence of LPS with the stability of the microbeads varying with the intensity of the inflammatory challenge (McQuilling et al., 2011; Khanna et al., 2013; Pareta et al., 2014; Appel et al., 2016; Ibarra et al., 2016; Brown et al., 2020; Shrestha et al., 2020) In the future it would be valuable to compare the stability of the microbeads upon exposure to a heightened inflammatory challenge, as observed in this study, to the native inflammatory response in the absence of LPS. The integrity of the LVG and methacrylated LVG outer layers were evaluated at 1 and 3 weeks. After 1 week, both groups had microbeads that remained intact with the microbeads visible in histological sections. The microbeads also remained intact through 3 weeks postimplantation. Inflammatory tissue surrounded the microbeads, including foreign body giant cells visible near the surface of the implants. Masson's trichrome staining also revealed a thin layer of collagen surrounding the microbeads. Quantitative analysis of the outer layer showed a decrease in outer layer size for the LVG group at 3 weeks as compared to preimplantation and 1 week. There was no statistical change in outer layer size for the methacrylated LVG group, showing that it was more stable than the non-methacrylated LVG group. These early time points may not be sufficient to comprehensively evaluate the improved stability but give insight into the potential of this method. A previous experiment conducted by our group compared single layered crude alginate microbeads to single layer methacrylated alginate beads using a similar LPS challenge. Nonmethacrylated microbeads were implanted into the omentum site and had completely failed by 1 week. (Somo et al., 2018) Similar results were not observed in this study. Though the in vitro results showed improved stability with methacrylation of the outer layer because microbeads both with and without a methacrylated outer layer remained intact up through 3 weeks, the in vivo results are inconclusive. In addition, the analysis methods are limited due to the results of histological processing on bead structure.

While the reason that the non-methacrylated beads survived longer in vivo as compared to previous studies is not known, changes in the microbead size and type of alginate used to stabilize the outer layer may have been a factor. Previous literature has shown that, when implanted, larger sized microbeads $>1,500 \mu \mathrm{m}$ elicit an attenuated foreign body reaction response compared to smaller microbeads (Veiseh et al., 2015). This may have been a factor even with an LPS challenge for LVG outer layer microbeads. LPS induces an immediate inflammatory response that is present regardless of the material implanted. When the acute inflammation caused by the LPS does not endure as long, there is less persistent inflammatory tissue (neutrophils, macrophages, and pro inflammatory mediators) that can further break down the outer layer and possibly invade within the inner layer. Another possible factor is the purity and type of alginate used. The di-axial configuration of the G-groups of the LVG allow for a stronger interaction with the cation. The alginate used to coat the outer layer consisted of $60 \%$ guluronic acid units. As described by the egg-box model, when sufficient cations are present, the outer layer created using LVG alginate would have increased mechanical strength (Gombotz, 1998). The alginate used in previous studies consisted of crude alginate, where the number of guluronic and mannuronic groups are unknown and can have a wide variation. This crude alginate was more likely to break down when exposed to LPS.

Microbeads can become destabilized by mechanical stresses and inflammation, resulting in swelling and degradation. While the exact mechanism of failure is unknown, stabilizing the outer layer can potentially prevent failure of implants. In this study, the outer LVG layer of A-PLO-A microbeads was methacrylated for covalent crosslinking as well as ionically crosslinked with calcium, which created a stable barrier to inflammatory intrusion. The microbeads were tested in vivo using an LPS challenge omentum pouch model. Beads with a methacrylated LVG outer layer were more stable than beads without under an inflammatory challenge, showing no significance decrease in outer layer size. Covalent crosslinking of the outer layer may be an important addition to cell encapsulation protocols to enhance stability. However, further studies are needed to examine long term survival and function.

\section{DATA AVAILABILITY STATEMENT}

The raw data supporting the conclusions of this article will be made available by the authors, without undue reservation.

\section{ETHICS STATEMENT}

The animal study was reviewed and approved by Institutional Animal Care and Use Committee of the Illinois Institute of Technology.

\section{AUTHOR CONTRIBUTIONS}

SS carried out the experiments, designed studies, interpreted data, wrote the paper, and edited the final document. EB designed studies, interpreted data, wrote the paper, and edited the final document. JB carried out additional experiments, performed data analysis, took the lead in writing the paper, and edited the final document. All authors contributed to the article and approved the submitted version.

\section{FUNDING}

This work was supported, in part, by funding from the National Institutes of Health (Grant No. 5R01EB020604) and the National Science Foundation (CBET-1263994, IIS-1125412). 


\section{REFERENCES}

Anderson, J. M., Rodriguez, A., and Chang, T. D. (2008). Foreign body reaction to biomaterials. Semin. Immunol. 20, 86-100. doi: 10.1016/j.smim.2007.11.004

Appel, A. A., Ibarra, V., Somo, S. I., Larson, J. C., Garson, A. B. III, Guan, H., et al. (2016). Imaging of hydrogel microsphere structure and foreign body response based on endogenous X-ray phase contrast. Tissue Eng. C Methods 22, 1038-1048. doi: 10.1089/ten.tec.2016.0253

Bhujbal, S. V., Paredes-Juarez, G. A., Niclou, S. P., and de Vos, P. (2014). Factors influencing the mechanical stability of alginate beads applicable for immunoisolation of mammalian cells. J. Mech. Behav. Biomed. Mater. 37, 196-208. doi: 10.1016/j.jmbbm.2014.05.020

Breger, J. C., Fisher, B., Samy, R., Pollack, S., Wang, N. S., and Isayeva, I. (2015). Synthesis of click alginate hydrogel capsules and comparison of their stability, water swelling, and diffusion properties with that of $\mathrm{Ca}(+2)$ crosslinked alginate capsules. J. Biomed. Mater. Res. B Appl. Biomater. 103, 1120-1132. doi: $10.1002 / j b m . b .33282$

Brown, J., Somo, S., Brooks, F., Komarov, S., Zhou, W., Anastasio, M., et al. (2020). X-ray CT in phase contrast enhancement geometry of alginate microbeads in a whole-animal model. Ann. Biomed. Eng. 48, 1016-1024. doi: 10.1007/s10439-019-02291-4

Calafiore, R., Basta, G., Luca, G., Lemmi, A., Montanucci, M. P., Calabrese, G., et al. (2005). Microencapsulated pancreatic islet allografts into nonimmunosuppressed patients with type 1 diabetes: first two cases. Diabetes Care 29, 137-138. doi: 10.2337/diacare.29.01.06.dc05-1270

Chandy, T., Mooradian, D. L., and Rao, H. G. (1999). Evaluation of modified alginate-chitosan-polyethylene glycol microcapsules for cell encapsulation. Artif. Organs. 23, 894-903. doi: 10.1046/j.1525-1594.1999.06244.x

de Vos, P., Smedema, I., van Goor, H., Moes, H., van Zanten, J., Netters, S., et al. (2003a). Association between macrophage activation and function of micro-encapsulated rat islets. Diabetologia 46, 666-673. doi: $10.1007 / \mathrm{s} 00125-003-1087-7$

de Vos, P., van Hoogmoed, C. G., van Zanten, J., Netter, S., Strubbe, J. H., and Busscher, J. H. (2003b). Long-term biocompatibility, chemistry, and function of microencapsulated pancreatic islets. Biomaterials 24, 305-312. doi: $10.1016 /$ S0142-9612(02)00319-8

Dinnyes, A., Schnur, A., Muenthaisong, S., Bartenstein, P., Burcez, C. T., Burton, N., et al. (2020). Integration of nano- and biotechnology for beta-cell and islet transplantation in type-1 diabetes treatment. Cell. Prolif. 53:e12785. doi: 10.1111/cpr.12785

Dufrane, D., Steenberghe, M., Goebbels, R. M., Saliez, A., Guiot, Y., and Gianello, P. (2006). The influence of implantation site on the biocompatibility and survival of alginate encapsulated pig islets in rats. Biomaterials 27, 3201-3208. doi: 10.1016/j.biomaterials.2006.01.028

Eiselt, P., Lee, K. Y., and Mooney, J. D. (1999). Rigidity of two-component hydrogels prepared from alginate and poly(ethylene glycol)-diamines. Macromolecules 32, 5561-5566. doi: 10.1021/ma990514m

Gombotz, W. (1998). Protein release from alginate matrices. Adv. Drug Deliv. Rev. 31, 267-285. doi: 10.1016/S0169-409X(97)00124-5

Hall, K. K., Gattas-Asfura, K. M., and Stabler, L. C. (2011). Microencapsulation of islets within alginate/poly(ethylene glycol) gels cross-linked via staudinger ligation. Acta Biomater. 7, 614-624. doi: 10.1016/j.actbio.2010.07.016

Hillberg, A. L., Oudshoorn, M., Lam, J. B., and Kathirgamanathan, K. (2015). Encapsulation of porcine pancreatic islets within an immunoprotective capsule comprising methacrylated glycol chitosan and alginate. J. Biomed. Mater. Res. B Appl. Biomater. 103, 503-518. doi: 10.1002/jbm.b.33185

Ibarra, V., Appel, A. A., Anastasio, M. A., Opara, E. C., and Brey, M. E. (2016). This paper is a winner in the undergraduate category for the SFB awards: evaluation of the tissue response to alginate encapsulated islets in an omentum pouch model. J. Biomed. Mater. Res. A 104, 1581-1590. doi: 10.1002/jbm.a.35769

Khanna, O., Huang, J. J., Moya, M. L., Wu, C. W., Cheng, M. H., Opara, E. C., et al. (2013). FGF-1 delivery from multilayer alginate microbeads stimulates a rapid and persistent increase in vascular density. Microvasc. Res. 90, 23-29. doi: 10.1016/j.mvr.2013.08.006

Khanna, O., Moya, M. L., Opara, E. C., and Brey, M. E. (2010). Synthesis of multilayered alginate microcapsules for the sustained release of fibroblast growth factor-1. J. Biomed. Mater. Res. A 95, 632-640. doi: 10.1002/jbm.a.32883
Kollmer, M., Appel, A. A., Somo, S. I., and Brey, M. E. (2016). Longterm function of alginate-encapsulated islets. Tissue Eng. B Rev. 22, 34-46. doi: 10.1089/ten.teb.2015.0140

Lawandi, J., Tao, C., Ren, B., Williams, P., Ling, D., Swan, M. A., et al. (2015). Reversal of diabetes following transplantation of an insulin-secreting human liver cell line: melligen cells. Mol. Ther. Methods Clin. Dev. 2:15011. doi: $10.1038 / \mathrm{mtm} .2015 .11$

Lee, K. Y., Rowley, J. A., Eiselt, P., Moy, E. M., Bouhadir, K. H., and Mooney, J. D. (2000). Controlling mechanical and swelling properties of alginate hydrogels independently by cross-linker type and cross-linking density. Macromolecules 33, 4291-4294. doi: 10.1021/ma9921347

Litwak, L., Goh, S. Y., Hussein, Z., Malek, R., Prusty, V., and Khamseh, E. M. (2013). Prevalence of diabetes complications in people with type 2 diabetes mellitus and its association with baseline characteristics in the multinational A1chieve study. Diabetol. Metab. Syndr. 5:57. doi: 10.1186/1758-5996-5-57

Mahou, R., Tran, N. M., Dufresne, M., Legallais, C., and Wandrey, C. (2012). Encapsulation of Huh-7 cells within alginate-poly(ethylene glycol) hybrid microspheres. J. Mater. Sci. Mater. Med. 23, 171-179. doi: $10.1007 /$ s10856-011-4512-3

McQuilling, J. P., Arenas-Herrera, J., Childers, C., Pareta, R. A., Khanna, O., Jiang, B., et al. (2011). New alginate microcapsule system for angiogenic protein delivery and immunoisolation of islets for transplantation in the rat omentum pouch. Transplant. Proc. 43, 3262-3264. doi: $10.1016 /$ j.transproceed.2011.10.030

Moya, M. L., Morley, M., Khanna, O., Opara, E. C., and Brey, M. E. (2012). Stability of alginate microbead properties in vitro. J. Mater. Sci. Mater. Med. 23, 903-912. doi: $10.1007 /$ s10856-012-4575-9

Omer, A., Duvivier-Kali, V. F., Trivedi, N., Wilmot, K., Bonner-Weir, S., and Weir, C. G. (2003). Survival and maturation of microencapsulated porcine neonatal pancreatic cell clusters transplanted into immunocompetent diabetic mice. Diabetes 52, 69-75. doi: 10.2337/diabetes.52.1.69

Pareta, R., McQuilling, J. P., Sittadjody, S., Jenkins, R., Bowden, S., Orlando, G., et al. (2014). Long-term function of islets encapsulated in a redesigned alginate microcapsule construct in omentum pouches of immune-competent diabetic rats. Pancreas 43, 605-613. doi: 10.1097/MPA.0000000000000107

Ponce, S., Orive, G., Hernandez, R., Gascon, A. R., Pedraz, J. L., de Haan, B. J., et al. (2006). Chemistry and the biological response against immunoisolating alginate-polycation capsules of different composition. Biomaterials 27, 4831-4839. doi: 10.1016/j.biomaterials.2006.05.014

Qi, M., Strand, B. L., Morch, Y., Lacik, I., Wang, Y., Salehi, P., et al. (2008). Encapsulation of human islets in novel inhomogeneous alginateca2+/ba2+ microbeads: in vitro and in vivo function. Artif. Cells Blood Substit. Immobil. Biotechnol. 36, 403-420. doi: 10.1080/107311908023 69755

Rengifo, H. R., Giraldo, J. A., Labrada, I., and Stabler, L. C. (2014). Long-term survival of allograft murine islets coated via covalently stabilized polymers. $A d v$. Healthc. Mater. 3, 1061-1070. doi: 10.1002/adhm.201300573

Sawhney, A. S., and Hubbell, J. A. (1992). Poly(ethylene oxide)-graftpoly(L-lysine) copolymers to enhance the biocompatibility of poly $(\mathrm{L}$ lysine)-alginate microcapsule membranes. Biomaterials 13, 863-870. doi: 10.1016/0142-9612(92)90180-V

Scharp, D. W., and Marchetti, P. (2014). Encapsulated islets for diabetes therapy: history, current progress, and critical issues requiring solution. Adv. Drug Deliv. Rev. 67-68, 35-73. doi: 10.1016/j.addr.2013.07.018

Shrestha, B., Stojkova, K., Yi, R., Anastasio, M. A., Ye, J. Y., and Brey, M. E. (2020). Gold nanorods enable noninvasive longitudinal monitoring of hydrogels in vivo with photoacoustic tomography. Acta Biomater. 7061, 30571-7. doi: 10.1016/j.actbio.2020.09.048

Somo, S. I., Khanna, O., and Brey, M. E. (2017). Alginate microbeads for cell and protein delivery. Methods Mol. Biol. 1479, 217-224. doi: 10.1007/978-1-4939-6364-5_17

Somo, S. I., Langert, K., Yang, C. Y., Vaicik, M. K., Ibarra, V., Appel, A. A., et al. (2018). Synthesis and evaluation of dual crosslinked alginate microbeads. Acta Biomater. 65, 53-65. doi: 10.1016/j.actbio.2017.10.046

Strand, B. L., Coron, A. E., and Skjak-Braek, G. (2017). Current and future perspectives on alginate encapsulated pancreatic islet. Stem Cells Transl. Med. 6, 1053-1058. doi: 10.1002/sctm.16-0116 
Thu, B., Bruheim, P., Espevik, T., Smidsrød, O., Soon-Shiong, P., and Skjåk-Bræk, G. (1996). Alginate polycation microcapsules. Biomaterials 17, 1031-1040. doi: 10.1016/0142-9612(96)84680-1

van Raamsdonk, J. M., Cornelius, R. M., Brash, J. L., and Chang, L. P. (2002). Deterioration of polyamino acid-coated alginate microcapsules in vivo. J. Biomater. Sci. Polym. Ed. 13, 863-884. doi: 10.1163/1568562023204 01933

Vandenbossche, G. M., Bracke, M. E., Cuvelier, C. A., Bortier, H. E., Mareel, M. M., and Remon, P. J. (1993). Host reaction against empty alginate-polylysine microcapsules. Influence of preparation procedure. J. Pharm. Pharmacol. 45, 115-120. doi: 10.1111/j.2042-7158.1993.tb03694.x

Vegas, A. J., Veiseh, O., Gurtler, M., Millman, J. R., Pagliuca, F. W., Bader, A. R., et al. (2016). Long-term glycemic control using polymer-encapsulated human stem cell-derived beta cells in immune-competent mice. Nat. Med. 22, 306-311. doi: $10.1038 / \mathrm{nm} .4030$
Veiseh, O., Doloff, J. C., Ma, M., Vegas, A. J., Tam, H. H., Bader, A. R., et al. (2015). Size- and shape-dependent foreign body immune response to materials implanted in rodents and non-human primates. Nat. Mater. 14, 643-651. doi: $10.1038 /$ nmat 4290

Conflict of Interest: The authors declare that the research was conducted in the absence of any commercial or financial relationships that could be construed as a potential conflict of interest.

Copyright $\odot 2020$ Somo, Brown and Brey. This is an open-access article distributed under the terms of the Creative Commons Attribution License (CC BY). The use, distribution or reproduction in other forums is permitted, provided the original author(s) and the copyright owner(s) are credited and that the original publication in this journal is cited, in accordance with accepted academic practice. No use, distribution or reproduction is permitted which does not comply with these terms. 